\title{
Ensayos OMA y calibración del MEF en el Sitio Arqueológico de Choq'epukyo-Perú (*)
}

\author{
S. Sovero ${ }^{1}$, R. Aguilar ${ }^{2}$, C. Martel $^{3}$
}

1 Docente contratada a tiempo completo en la Facultad de Ingeniería y Arquitectura, E.P. Ingeniería Civil de la Universidad Andina del Cusco. Urb. Ingeniería, Larapa Grande A-5. San Jerónimo. Cusco Perú.

2 Profesor asociado del Departamento de Ingeniería de la Pontificia Universidad Católica del Perú. Av. Universitaria 1801, San Miguel, Lima, Perú

3 Magister Egresada del Departamento de Ingeniería de la Pontificia Universidad Católica del Perú. Av. Universitaria 1801, San Miguel, Lima, Perú

e-mail: s.sovero@uandina.edu.pe, raguilar@pucp.edu.pe, carol.martel@pucp.edu.pe

(*) Articulo presentado en el Congreso EVACES, 2013

\section{RESUMEN}

La conservación de estructuras históricas es de suma importancia para la preservación del patrimonio cultural de la humanidad. La comprensión del comportamiento estructural de este tipo de construcciones es, particularmente compleja, debido a la dificultad que existe para obtener una adecuada caracterización de los materiales, las condiciones de borde, y el estado de daño que puedan presentar. En este artículo se llevaron a cabo, ensayos modales vibracionales (OMA), para estudiar un muro preinca de albañilería de piedra que forma parte del sitio arqueológico de Choq'epukyo, ubicado en Cusco, Perú ( 1100 d. D.). La estructura estudiada tiene $18 \mathrm{~m}$ de longitud y $9 \mathrm{~m}$ de altura y está hecho de piedra irregular y mortero de barro con unidades en una configuración multicapa. Este artículo presenta a detalle los resultados de los ensayos, el procesamiento de datos usando el método de identificación del subespacio estocástico. El artículo también presenta el análisis de sensibilidad llevado a cabo en el modelo de elementos finitos y el uso de un algoritmo de optimización.

Palabras clave: Análisis modal operacional, sitios arqueológicos, albañilería de piedra, modelo numérico de elementos finitos, calibración de modelos. 


\begin{abstract}
The conservation of historical structures is of high importance for preserving the cultural heritage of humanity. The assessment of the structural behaviour of these types of buildings is particularly complex due to the difficulty of an accurate characterization of materials, boundary conditions, and damage state. In this paper, OMA tests are used to study a Pre-Inca stone masonry wall, which is part of the archaeological site of Choq'epukyo in Cusco, Perú ( 1100 A.D). The studied structure has $18 \mathrm{~m}$ length and $9 \mathrm{~m}$ height, and is made of irregular stone and mud mortar with bricks in multileaf configuration. The paper presents the details of the tests and the results of the data processing stage, which was performed using the stochastic subspace identification method. The paper also discusses the process of finite element model calibration which was carried out by means of a sensitivity analysis and the use of optimization algorithms.
\end{abstract}

Keywords: Operational modal analysis, archaeological sites, stone masonry, numerical model of finite elements, calibration of models. 


\section{INTRODUCCIÓN}

La conservación y mantenimiento de las estructuras históricas es de gran importancia para salvaguardar el patrimonio cultural de la humanidad. Comprender el comportamiento estructural de este tipo de construcciones históricas es, en especial, complejo debido a la dificultad que existe para caracterizar la geometría, los materiales, el estado de daño; para identificar el sistema estructural, así como para elaborar un modelo numérico confiable (Lourenḉo, 2006). El Concilio Internacional de Sitios y Monumentos (ICOMOS), organización responsable de la conservación del patrimonio cultural, establece diferentes estrategias para el estudio de construcciones históricas. Estas estrategias consisten en realizar campañas extensas y exhaustivas basadas en ensayos no destructivos, así como, investigaciones in situ y en laboratorio (Roca et al., 2010). En este contexto, los ensayos no destructivos se convierten en una herramienta importante, ya que permiten realizar una evaluación de las construcciones históricas sin causarles daño. El análisis vibracional experimental, conocido como Análisis Modal Operacional (OMA), es una de las más poderosas técnicas que permiten identificar los parámetros dinámicos de la estructura. Con estos resultados experimentales, se podrá realizar la calibración de un «modelo numérico de elementos finitos» (FEM).

En el presente estudio se ha llevado a cabo, un ensayo experimental OMA en uno de los últimos vestigios del Sitio Arqueológico de Choq'epukyo (Academia Mayor de la Lengua Quechua, 2005), en Cusco, Perú. Los resultados de estos ensayos experimentales fueron usados para calibrar el FEM de la estructura, lo cual significa el primer paso para estudiar la vulnerabilidad sísmica de la construcción.

\section{EL SITIO ARQUEOLÓGICO DE CHOQ'EPUKYO}

El Sitio Arqueológico de Choq'epukyo está ubicado a $30 \mathrm{~km}$ de la ciudad del Cusco (Perú), donde una gran variedad de restos construidos con albañilería de piedra y mortero de barro se encuentran en este lugar (ver figura 1). Es de lamentar, que debido al paso del tiempo y a la ocurrencia de sismos (Perú está localizado en una de las zonas sísmicas más activas del mundo), este centro arqueológico esté severamente dañado. Los mayores daños consisten en la pérdida de unidades de piedra y del recubrimiento debido a las duras condiciones climáticas y presencia de vegetación. 


\section{Descripción histórica}

Después de la desaparición de la cultura Wari, y antes del imperio incaico, una pequeña cultura conocida como la «Cultura Lucre», se desarrolló en la zona de Choq'epukyo, Cusco. Se dice que este asentamiento fue construido entre los años 900 a 1300 d.C., y es considerado como el lugar principal donde se centró el ‘período intermedio tardío’ de la historia peruana. En Choq'epukyo se encuentran nichos para rituales y festividades, los cuales nos sindican que este centro arqueológico tenía propósitos religiosos (McEwan et al. 2005). Este lugar antiguo está ubicado en el área donde confluyen dos ríos importantes: el Huatanay y el Vilcanota. Esto - y la presencia de reservorios y canales que cruzan los recintos-, es una evidencia de que los ocupantes rendían culto al agua. Sumado a todo esto, existen vestigios de muros y fundaciones que nos dan una idea del tamaño que tenía Choq'epukyo en su tiempo.

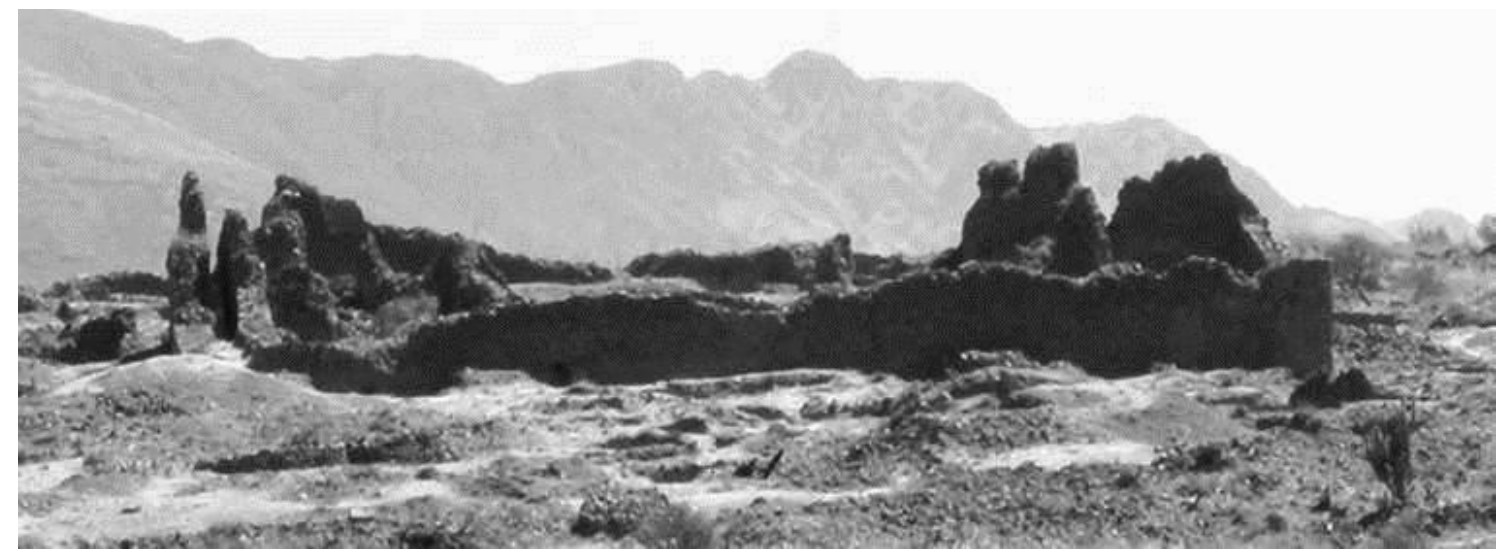

Figura 1: Vista Panorámica de un sector del Sitio Arqueológico de Choq’epukyo

\section{Descripción arquitectónica}

El Sitio Arqueológico de Choq'epukyo presenta una arquitectura especial con muros que encierran espacios a cielo abierto. Con esta configuración se pueden observar: calles y pasillos angostos que conectan los accesos de dichos espacios. En la actualidad, se han encontrado vestigios que muestran alrededor de diez ambientes (llamados «kanchas»), que corresponden a un asentamiento urbano. En general, los muros perimetrales tienen 12 metros de altura y contienen nichos (ventanas cerradas) trapezoidales y rectangulares a diferentes 
alturas. La albañilería “rústica” y “simple” encontrada en Choq’epukyo está hecha con piedra semiredondeada y juntas irregulares de barro y paja. En algunos casos, el recubrimiento de barro original es aún visible en los muros y en los nichos (McEwan et al. 2005).

\section{Descripción estructural}

El Sitio Arqueológico de Choq'epukyo fue construido con material de la zona, compuesto principalmente por piedra andesita. El sistema de albañilería está mezclado por piedras irregulares y juntas de mortero cuyo espesor varía desde 2,5 a 10 centímetros. La altura de estas estructuras también es variable, entre 10 y 12 metros. El área de estudio elegida es un sector llamado «Área A». En la figura 2, se muestra una vista en planta del área estudiada. Como se puede ver, la evaluación estructural se llevó a cabo en una zona localizada en la esquina de dicho sector que consiste de dos muros conectados entre ellos, por medio de puntales de madera. Este sector fue seleccionado como caso de estudio debido a su buen estado de preservación (el revestimiento original se puede observar en la cara de ambos muros, ver figura 2d). Para este estudio solo el muro frontal fue instrumentado, puesto que no tiene conexión estructural con otros muros. Esta pared, que tiene una geometría irregular, posee más de $18 \mathrm{~m}$ de longitud y $9 \mathrm{~m}$ de altura, con espesor variable, que va desde 1,20 m, a $1,80 \mathrm{~m}$, en la base hasta $0,60 \mathrm{~m}$, en la punta (ver figuras $2 \mathrm{~b}$ y $2 \mathrm{c}$ ).

La diferencia en la disposición de la albañilería de piedra usada (entre la base y la punta), es una evidencia de que existen dos diferentes calidades de albañilería. En la base, se observa una albañilería más consolidada y ordenada y fue construida usando piedras grandes asentadas con mortero de barro. Por otro lado, la parte alta el muro fue fabricada con piedras más pequeñas y juntas de mortero de barro más gruesas. 


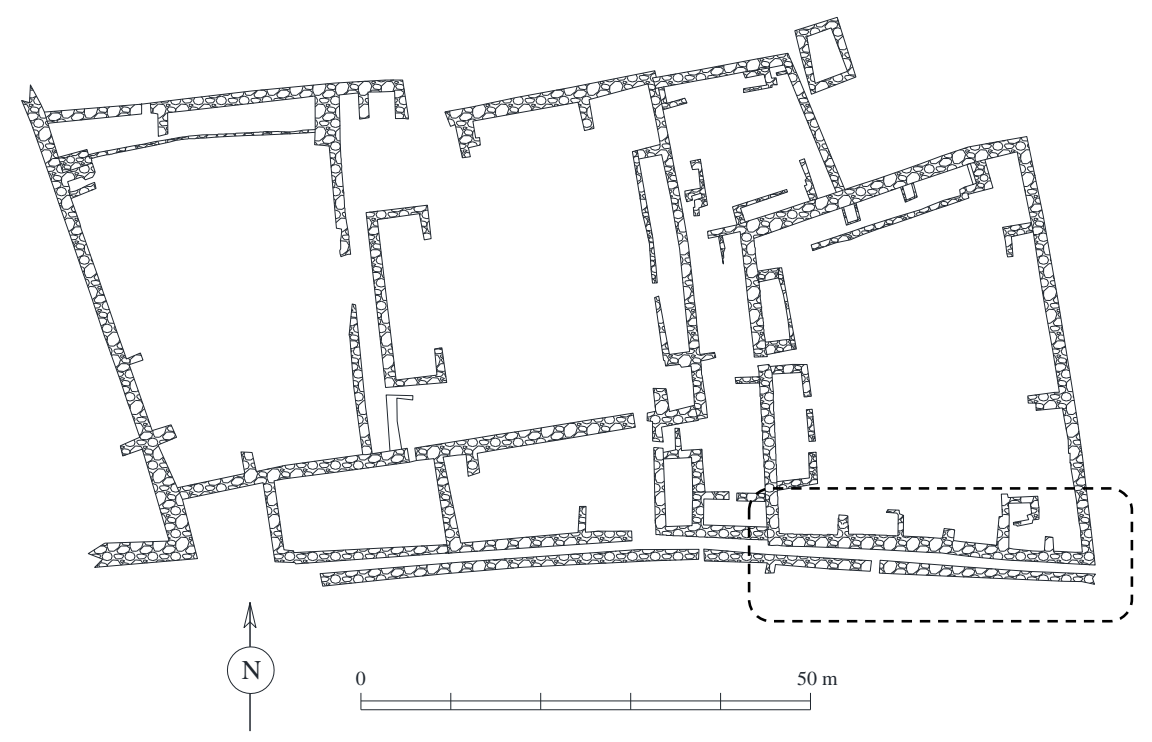

(a)

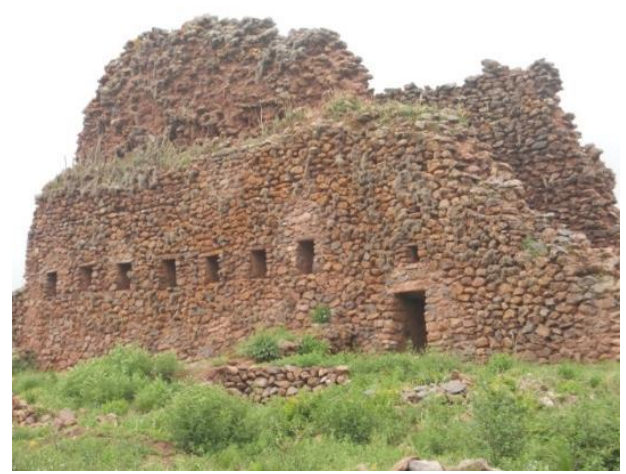

(b)

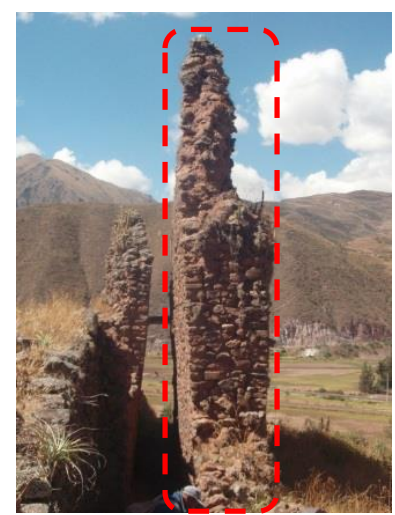

(c)

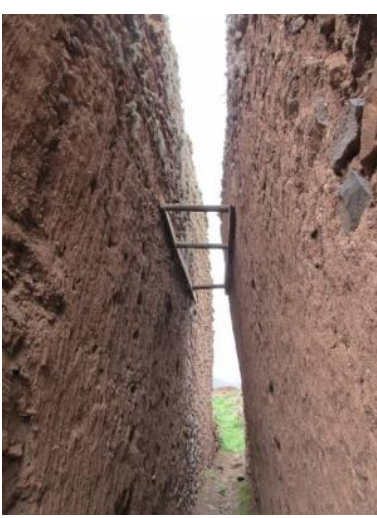

(d)

Figura 2 : Vista general del área de estudio: (a) vista en planta del sector en estudio (McEwan et al. 2005); (b) vista en elevación del muro instrumentado, (c) vista lateral del muro instrumentado; y (d) detalle del puntal de madera colocado entre el muro instrumentado y el muro posterior

Las patologías encontradas en la zona de estudio se deben: a la vegetación, al paso del tiempo y a la ocurrencia de sismos que han causado deterioro en la base y en la parte alta del paramento. El muro estudio presenta, además, una inclinación que ha sido controlada colocando tres puntales de madera que conectan el muro estudio con el muro posterior. La interacción entre estos dos paramentos se observa en las figuras 2c y 2 d.

\section{Ensayo modal operacional}

Con el objetivo de identificar la respuesta dinámica del muro en estudio, se llevó a cabo un ensayo experimental y utilizamos ruido ambiental como fuente de excitación de la estructura. Este ensayo experimental se desarrolló en el mes de diciembre del año 2012. Dieciséis puntos 
de medición se establecieron para adquirir el mayor número de datos posible. Los transductores fueron: cuatro acelerómetros piezoeléctricos, con una sensibilidad $10 \mathrm{~V} / \mathrm{g}$ y un rango dinámico de $\pm 0,5 \mathrm{~g}$; junto a un sistema de adquisición de datos de 24 bits de resolución, con entrada $U S B$ de 24 bits. Fue necesario colocar un sistema de andamios (no conectado a la estructura) para poder ubicar los transductores a lo largo del muro (tal como se muestra en la figura 3a). Adicionalmente, se midieron las vibraciones del suelo para poder estudiar la interacción suelo-estructura en futuras investigaciones.

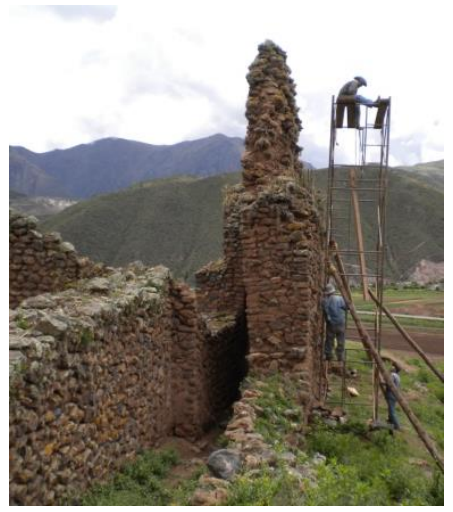

(a)

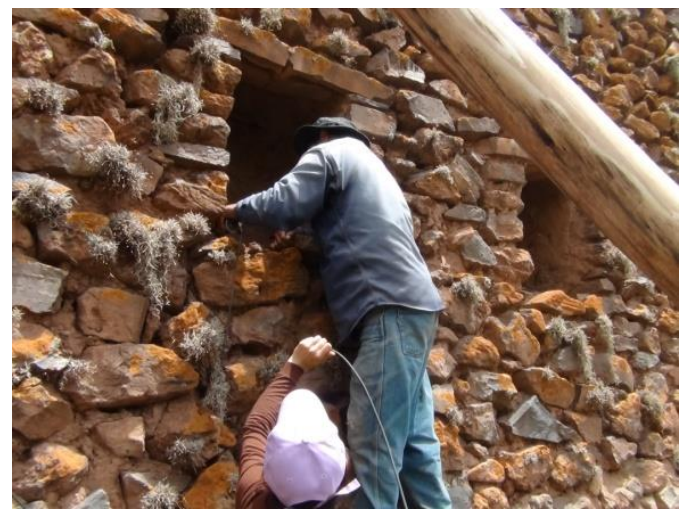

(b)

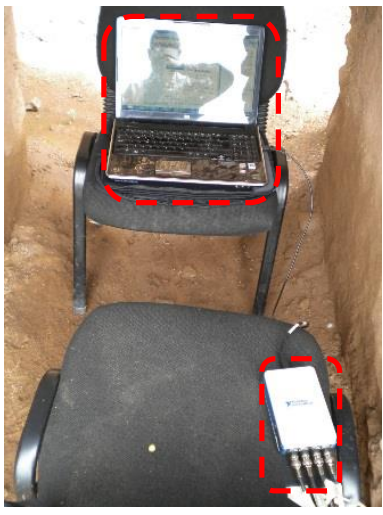

(c)

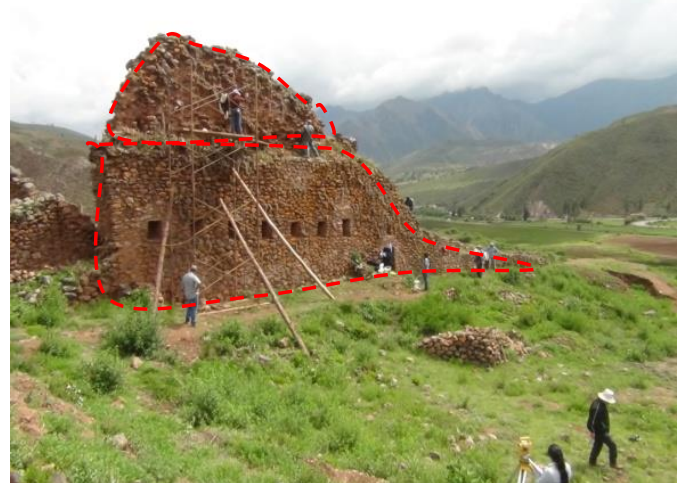

(d)

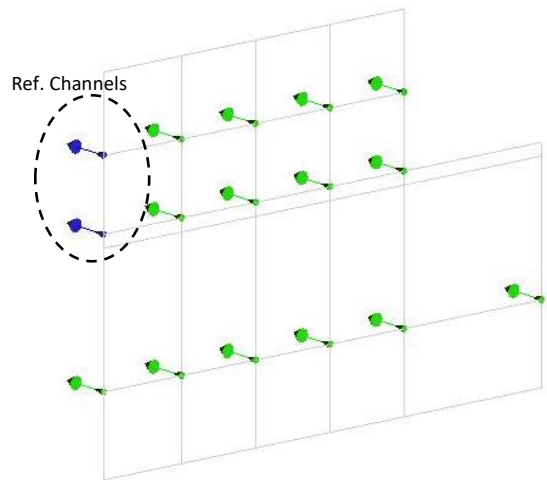

(e)

Figura 3: Ensayos OMA en el sitio arqueológico de Choq'epukyo: (a) y (b) ubicación de los sensores en el muro; (c) sistema de adquisición de datos; (d) y (e) disposición de los puntos instrumentados.

La identificación de la respuesta dinámica se realizó usando técnicas de procesamiento de señales en el dominio del tiempo — a través del método SSI implementado en el software ARTEMIS (SVS, 2013) —. El diagrama resultante de la aplicación del método se muestra en la figura 4. Como se expone, se han podido identificar, al menos, cuatro arreglos de puntos alineados estables, que indican las cuatro primeras frecuencias naturales de la estructura. Por 
tal motivo, en el proceso de calibración del modelo numérico solo se actualizarán los cuatro primeros modos de la estructura.

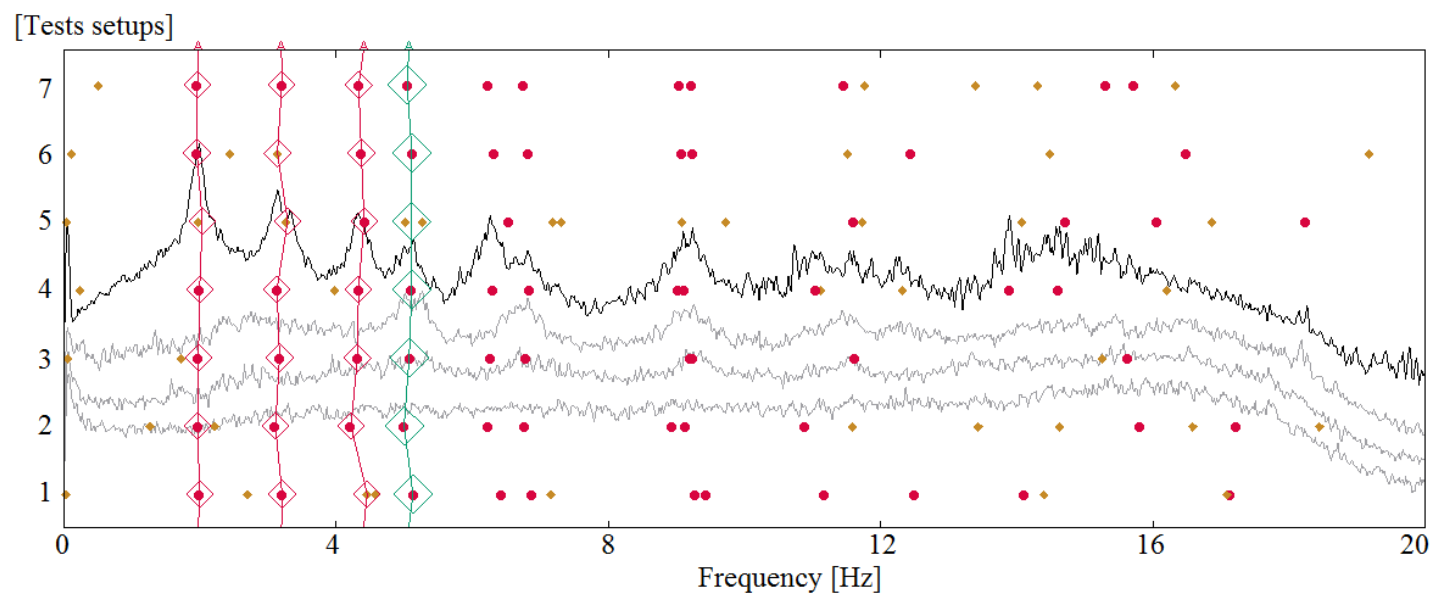

Figura 4 : Diagrama estabilizado después de la aplicación del método SSI, para el proceso de adquisición de datos.

El comportamiento dinámico del muro instrumentado, basado en sus cuatro primeras frecuencias naturales, ratios de amortiguamiento y formas modales se muestran en la figura 5.

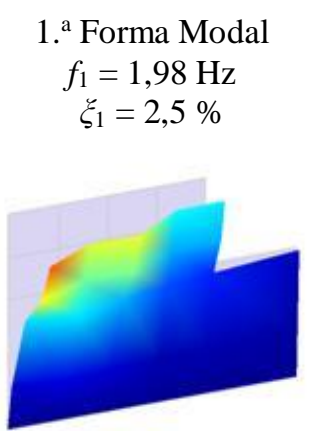

$$
\begin{gathered}
\text { 2. }{ }^{\text { Forma Modal }} \\
f_{2}=3,18 \mathrm{~Hz} \\
\xi_{2}=2,8 \%
\end{gathered}
$$

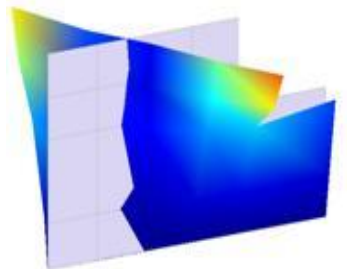

3. ${ }^{\mathrm{a}}$ Forma Modal

$f_{3}=4,33 \mathrm{~Hz}$

$\xi_{3}=2,3 \%$

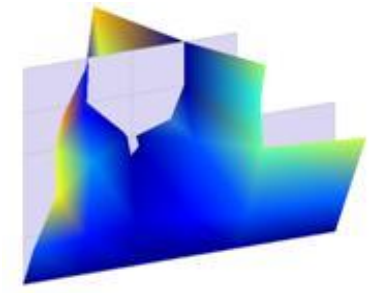

4. ${ }^{\mathrm{a}}$ Forma Modal

$f_{4}=5,02 \mathrm{~Hz}$

$\xi_{4}=1,6 \%$

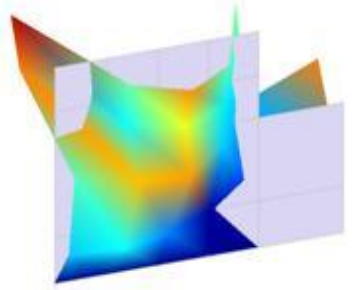

Figura 5 : Resultados de los ensayos experimentales en el sitio arqueológico de Choq'epukyo.

\section{MODELO NUMÉRICO Y PROCESO DE CALIBRACIÓN}

Varios modelos computacionales se desarrollaron usando el software SAP 2000 (CSI, 2013). Los modelos fueron realizados considerando elementos sólidos con propiedades homogéneas. Debido a que no se realizaron ensayos en laboratorio y, por lo tanto, las propiedades mecánicas del material eran desconocidas, se llevó a efecto una investigación bibliográfica para completar el FEM del muro. La tabla 1 muestra las propiedades mecánicas 
de albañilería de piedra similares. Para el presente estudio se eligieron como parámetros base los valores proporcionados por Brignola (2008).

Tabla 1: Propiedades mecánicas de estructuras de albañilería de piedra

\begin{tabular}{|c|c|c|c|}
\hline Descripción & Tipología de muros & $\begin{array}{c}\mathrm{E} \\
{[\mathrm{GPa}]}\end{array}$ & $\begin{array}{l}\text { Poisson } \\
\text { ratio }\end{array}$ \\
\hline Laboratory test (Porto et. al., 2003) & $\begin{array}{l}\text { Irregular stone with lime mortar, } \\
\text { sand }(1: 3) \text { and water }\end{array}$ & $0,3-5,1$ & \\
\hline In-situ test (Porto et. al., 2003) & Irregular stone with lime mortar & $0,1-1,3$ & \\
\hline Laboratory test (Brignola, 2008) & $\begin{array}{l}\text { Volcanic Stone with lime mortar and } \\
\text { sand }(e=50 \mathrm{~cm})\end{array}$ & 1,2 & 0,2 \\
\hline Laboratory test (Brignola, 2008) & Multi-leaf irregular stone lime mortar & 0,8 & 0,2 \\
\hline Laboratory test (Manos, 2008) & Stone with lime mortar & 2,5 & 0,2 \\
\hline Laboratory test (Almeida et al., 2011) & $\begin{array}{l}\text { Irregular stone with lime mortar and sand } \\
(1: 3)\end{array}$ & $0,2-0,3$ & $0,2-0,3$ \\
\hline
\end{tabular}

Con el objetivo de realizar un análisis de sensibilidad, se evaluaron tres sistemas estructurales del muro. El primer sistema se desarrolló al considerar, que existe una interacción entre el muro instrumentado y el muro sobre el cual se apoya (figura 6a). En este FEM, los puntales de madera fueron representados por elementos lineales hechos de madera eucalipto con un módulo de elasticidad de 5,4 GPa (tal como se establece en la NTE E.010, 2006). El segundo sistema estructural se desarrolló con una conexión parcial entre los dos muros. Para este FEM, los puntales se representaron como resortes con una rigidez axial equivalente (Figura 6b). Finalmente, el tercer sistema estructural se desarrolló considerando que no existe ninguna interacción entre los dos muros (Figura 6c). En el último modelo, solo se estudió el muro instrumentado. En todos los modelos el peso específico del sistema estructural se estableció como $23,5 \mathrm{kN} / \mathrm{m}^{3}$. 


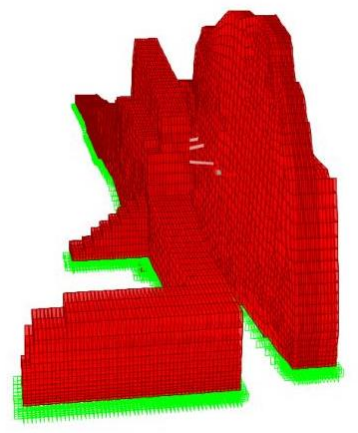

(a)

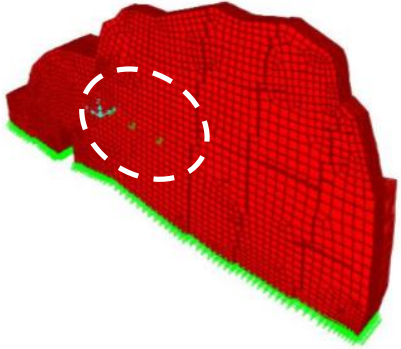

(b)

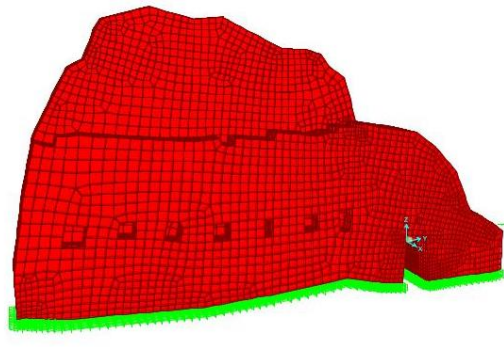

(c)

Figura 6 : Sistemas estructurales considerados: (a) primero: FEM considerando interacción completa; (b) segundo: FEM considerando interacción parcial; and (c) tercero: FEM considerando ninguna interacción entre los dos muros.

Las cuatro primeras frecuencias naturales y formas modales resultantes del FEM y de los ensayos experimentales, se compararon usando el 'Criterio de Exactitud Modal' (MAC). Este criterio establece una relación entre dos vectores modales cuyo valor varía en un rango de 0 a 1. Cuanto más se acerque el valor MAC a la unidad, significa que los resultados son más consistentes (Allemang, 2003). La expresión para calcular el valor MAC está definida por la ecuación (1):

$$
M A C=\frac{\left|\sum_{i=1}^{n} \varphi_{i}^{\exp } \varphi_{i}^{F E}\right|^{2}}{\sum_{i=1}^{n}\left(\varphi_{i}^{\exp }\right)^{2} \sum_{i=1}^{n}\left(\varphi_{i}^{F E}\right)^{2}}
$$

Donde $\varphi^{\text {exp }}$, y $\varphi^{\mathrm{FE}}$, son los vectores modales experimental y numérico, respectivamente.

\section{Resultados}

El resumen de los resultados expresados en frecuencias y valores MAC, calculados para los tres modelos, se presenta en la tabla 2. Como se puede observar, las frecuencias tienen una buena correlación entre los resultados experimentales y numéricos en todos los modelos. El máximo error relativo fue de $14 \%$ en el primer modelo, $19 \%$ en el segundo modelo, y $14 \%$ en el tercer modelo. Los valores MAC también muestran una buena correlación, aunque en la tercera y cuarta forma modal existe mayor variación. 
Tabla 2: Resultados de las frecuencias experimentales y numéricas para cada modelo

\begin{tabular}{lcccccccc}
\hline & $\begin{array}{c}\text { Experimental } \\
\text { Frecuencia } \\
{[\mathrm{Hz}]}\end{array}$ & $\begin{array}{c}1 .{ }^{\circ} \text { Modelo } \\
\text { Frecuencia } \\
{[\mathrm{Hz}]}\end{array}$ & MAC & $\begin{array}{c}2 .{ }^{\circ} \text { Modelo } \\
\text { Frecuencia } \\
{[\mathrm{Hz}]}\end{array}$ & MAC & $\begin{array}{c}3 .{ }^{\circ} \text { Modelo } \\
\text { Frecuencia } \\
{[\mathrm{Hz}]}\end{array}$ & MAC \\
\hline $1 .{ }^{\circ}$ Modo & 1,98 & 2,08 & {$[5 \%]$} & 0,99 & $1,95[2 \%]$ & 0,99 & $1,74[12 \%]$ & 0,99 \\
$2 .{ }^{\circ}$ Modo & 3,17 & 3,62 & {$[14 \%]$} & 0,96 & $3,78[19 \%]$ & 0,94 & $3,31[4 \%]$ & 0,97 \\
$3 .{ }^{\circ}$ Modo & 4,33 & 4,43 & {$[2 \%]$} & 0,72 & $4,78[10 \%]$ & 0,59 & $4,47[3 \%]$ & 0,80 \\
$4 .{ }^{\circ}$ Modo & 5,02 & 5,04 & {$[<1 \%]$} & 0,68 & $5,83[16 \%]$ & 0,79 & $5,73[14 \%]$ & 0,78 \\
\hline * (El error relativo se muestra entre corchetes) & & & & & &
\end{tabular}

Seguidamente se realizó un proceso de optimización, tal como lo propone Ramos et al. (2012), usando una función objetivo con la finalidad de minimizar la diferencia entre los resultados experimentales y numéricos. Este proceso de optimización se realizó solo en el tercer FEM, se ha considerado el hecho de que sus resultados numéricos son más cercanos a las formas modales obtenidas con los ensayos experimentales OMA (incluso si en frecuencias los valores tienen mayor incertidumbre). Para construir la función objetivo, se usó el enfoque propuesto por Douglas-Reid (1982). Para esto se predefinieron: valores base, valores tope bajos y valores tope altos. En este caso, para elegir dichos valores se realizó un análisis de sensibilidad con el fin de determinar la influencia de los parámetros mecánicos (módulo de elasticidad y peso específico), de las condiciones de borde y, la localización de los daños en los resultados dinámicos.

Los resultados del análisis de sensibilidad mostraron que los parámetros mecánicos eran los que más influían en los resultados, de tal manera que, el módulo de elasticidad afectaba de mayor manera los resultados de frecuencias naturales y el peso específico del material, del mismo modo, afectaba de mayor manera las formas modales del sistema. Con el objetivo de refinar el FEM y obtener mejores resultados del proceso de calibración, se modeló el muro en estudio, al considerar que está compuesto de dos materiales diferentes: uno en la base del muro y otro en la parte superior del muro (desde donde se observa el cambio de sección).

Las tablas 3 y 4 muestran el resumen del proceso de calibración. Tal como se puede observar, los valores finales de los parámetros que caracterizan el material (módulo de elasticidad y peso específico) confirman la diferencia que existe entre el material de la base y de la parte alta del muro en estudio. La alta correspondencia que existe entre los resultados 
experimentales también se evidencia claramente, ya que las frecuencias naturales y valores MAC presentan errores muy pequeños.

Tabla 3:Valores iniciales y finales después del proceso de calibración

\begin{tabular}{ccccc}
\hline Parámetros a calibrar & $\begin{array}{c}\mathrm{E}_{\text {base muro }} \\
{[\mathrm{MPa}]}\end{array}$ & $\begin{array}{c}\mathrm{E}_{\text {parte alta muro }} \\
{[\mathrm{MPa}]}\end{array}$ & $\begin{array}{c}\gamma \text { base muro } \\
{[\mathrm{N} / \mathrm{mm} 3]}\end{array}$ & $\begin{array}{c}\gamma \text { parte alta muro } \\
{[\mathrm{N} / \mathrm{mm} 3]}\end{array}$ \\
\hline Valores iniciales & 800.0 & 800.0 & $269.0 \mathrm{E}-3$ & $269.0 \mathrm{E}-3$ \\
Valores finales & 643,7 & 426,9 & $342,6 \mathrm{E}-3$ & $316,5 \mathrm{E}-3$ \\
\hline
\end{tabular}

Tabla 4: Comparación de los resultados del análisis del FEM calibrado con los resultados experimentales

\begin{tabular}{lcccc}
\hline & Experimental[Hz] & FEM[Hz] & Error & MAC \\
\hline 1. Modo & 1,98 & 1,82 & $7,8 \%$ & 1,00 \\
2. Modo & 3,17 & 3,42 & $8,0 \%$ & 0,98 \\
3. Modo & 4,33 & 4,33 & $<1 \%$ & 0,76 \\
4. Modo & 5,02 & 5,67 & $13,0 \%$ & 0,81
\end{tabular}

\section{CONCLUSIONES}

Esta investigación presenta al detalle el ensayo operacional modal aplicado en el Sitio Arqueológico de Choq'epukyo en Cusco Perú. La investigación tuvo como objetivo estudiar uno de los sectores del sitio que es importante para su adecuada preservación. Los resultados del ensayo OMA en el muro instrumentado muestran que las primeras cuatro frecuencias naturales fueron identificadas con una alta confiabilidad. Por otro lado, el FEM mostró que el muro puede ser estudiado como un sistema estructural simple, sin considerar la interacción con el muro sobre el cual se encuentra apoyado. Los resultados del proceso de calibración muestran que el FEM calibrado tiene alta correspondencia con respecto a los resultados obtenidos experimentalmente. Estos resultados son de gran importancia para llevar a cabo futuros estudios de evaluaciones de vulnerabilidad sísmica. 


\section{BIBLIOGRAFÍA}

Academia Mayor de la Lengua Quechua. (2005). Diccionario quechua, español, quechua (2.da Edición ed.). Cusco, Perú: Gobierno Regional Cusco.

Allemang , J R (2003). "The Modal Assurance Criterion - Twenty Years of Use and Abuse." Journal of Sound and Vibration, 14-21pp

Brignola A., Frumento S., Lagomarsino S., Podesta E. (2008) Identification of Shear Parameters of Masonry Panels Through the In-Situ Diagonal Compression Test, Journal of Architectural Heritage 3:1, 52-73pp

CSI. 2013 SAP2000, Computers and Structures Inc., USA

Douglas-Reid, B.M., W.H. (1982). Dynamic tests and system identification of bridges, Journal Struct. Div., ASCE, 108, 2295-2312

Lourenço P B. (2006) Recommendations for restoration of ancient buildings and the survival of a masonry chimney. Journal of Construction and Building Materials 20, 239-251pp

Manos G.C, Soulis V.J., Diagouma A. (2008) Numerical investigation of the behaviour of the church of Agia Triada, Drakotrypa, Greece. Journal of Advances in Engineering Software 39, 284-300pp

Mc Ewan, Gordon; Gibaja, Arminda; Chatfiel, Melisa. (2005) Arquitectura monumental en el Cuzco en el período Intermedio Tardío: Evidencia de Continuidades en la Reciprocidad Ritual y el Manejo Administrativo entre los Horizontes Medio y Tardío. Boletín de Arqueología PUCP.

Porto F, Valluzzi M.R., Modena C. (2003) Investigations for the knowledge of multi-Ieaf stone masonry walls. Proceedings of the first International Congress on Construction History, Madrid, 20th-24th January 2003

NTE E.010, 2006, Norma Técnica de Edificación, Madera, Ed. SENCICO

Ramos, L, Aguilar, R., Lourenco, P., Moreira, S. (2012) "Dynamic structural health monitoring of Saint Torcato church", Journal of Mechanical Systems and Signal Processing

SVS. 2013 ARTEMIS Extractor Pro User Manual. Structural Vibration Solutions, Aalborg, Denmark 